\title{
Evaluation of adjuvant activity of fractions derived from Agaricus blazei, when in association with the recombinant LiHyp1 protein, to protect against visceral leishmaniasis
}

\author{
Nathália Cristina de Jesus Pereira a , Wiliam César Bento Régis ${ }^{b, c}$, \\ Lourena Emanuele Costa a , Jamil Silvano de Oliveira ${ }^{d}$, Alanna Gomes da Silva ${ }^{c}$, \\ Vivian Tamietti Martins ${ }^{\mathrm{d}}$, Mariana Costa Duarte ${ }^{\mathrm{a}, \mathrm{h}}$, José Roberto Rodrigues de Souza ${ }^{\mathrm{e}}$, \\ Paula Sousa Lage a , Mônica Santos Schneider ${ }^{\mathrm{a}}$, Maria Norma Melo ${ }^{\mathrm{f}}$, Manuel Soto ${ }^{\mathrm{g}}$, \\ Sandra Aguiar Soares e , Carlos Alberto Pereira Tavares ${ }^{\mathrm{d}}$, Miguel Angel Chávez-Fumagalli a, \\ Eduardo Antonio Ferraz Coelho a,h,* \\ a Programa de Pós-Graduação em Ciências da Saúde: Infectologia e Medicina Tropical, Faculdade de Medicina, Universidade Federal de Minas Gerais, \\ 30130-100 Belo Horizonte, Minas Gerais, Brazil \\ b Instituto de Ciências Biológicas e da Saúde, Pontifícia Universidade Católica de Minas Gerais, 30535-901 Belo Horizonte, Minas Gerais, Brazil \\ ' Minasfungi do Brasil Ltda, Belo Horizonte, Minas Gerais, Brazil \\ d Departamento de Bioquímica e Imunologia, Instituto de Ciências Biológicas, Universidade Federal de Minas Gerais, 31270-901 Belo Horizonte, Minas \\ Gerais, Brazil \\ e Departamento de Química Orgânica e Inorgânica, Centro de Ciências, Universidade Federal do Ceará, 60356-682 Fortaleza, Ceará, Brazil \\ f Departamento de Parasitologia, Instituto de Ciências Biológicas, Universidade Federal de Minas Gerais, $31270-901$ Belo Horizonte, Minas Gerais, Brazil \\ g Centro de Biología Molecular Severo Ochoa, CSIC-UAM, Departamento de Biología Molecular, Universidad Autónoma de Madrid, 28049 Madrid, Spain \\ h Departamento de Patologia Clínica, COLTEC, Universidade Federal de Minas Gerais, 31270-901 Belo Horizonte, Minas Gerais, Brazil
}

\section{H I G H L I G H T S}

- Purification of polysaccharide-rich fractions from Agaricus blazei mushroom.

- In vitro immune stimulation experiments using spleen cells of BALB/c mice.

- Selection of the two best adjuvant fractions (F2 and F4).

- Protection of LiHyp1 protein plus F2, F4 or saponin against Leishmania infantum.

- Identification of new Th1 immune response adjuvants derived from Agaricus blazei.
G R A P H I C A L A B S T R A C T

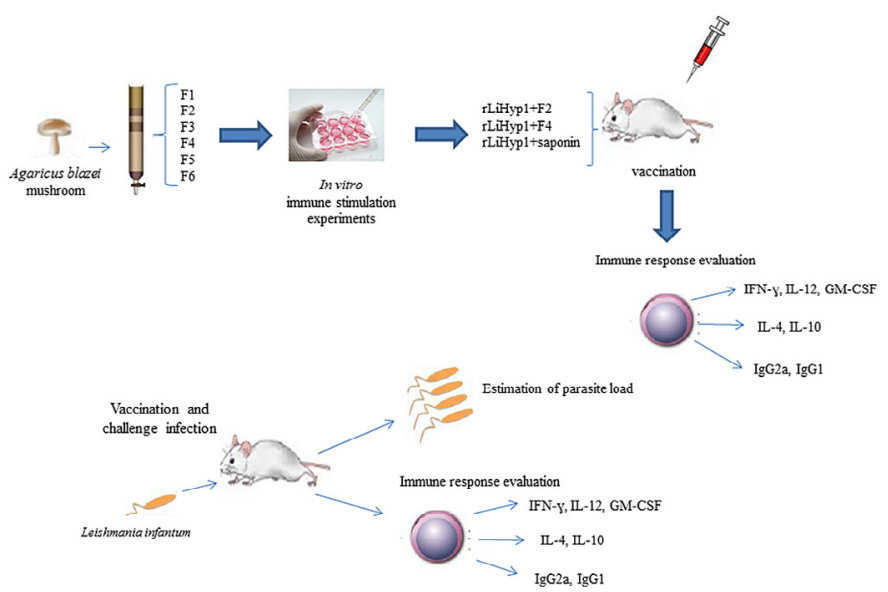

* Corresponding author. Fax: +55 3134094983.

E-mail address: eduardoferrazcoelho@yahoo.com.br (E.A.F. Coelho). 


\section{A R T I C L E I N F O}

Article history:

Received 23 October 2014

Received in revised form 23 March 2015

Accepted 25 March 2015

Available online 3 April 2015

\section{Keywords:}

Visceral leishmaniasis

Agaricus blazei

Polysaccharide-rich fractions

Th1 immune response

Adjuvants

BALB/c mice
A B S T R A C T

The development of effective prophylactic strategies to prevent leishmaniasis has become a high priority. No less important than the choice of an antigen, the association of an appropriate adjuvant is necessary to achieve a successful vaccination, as the majority of the tested antigens contain limited immunogenic properties, and need to be supplemented with immune response adjuvants in order to boost their immunogenicity. However, few effective adjuvants that can be used against leishmaniasis exist on the market today; therefore, it is possible to speculate that the research aiming to identify new adjuvants could be considered relevant. Recently, Agaricus blazei extracts have proved to be useful in enhancing the immune response to DNA vaccines against some diseases. This was based on the Th1 adjuvant activity of the polysaccharide-rich fractions from this mushroom. In this context, the present study evaluated purified fractions derived from Agaricus blazei as Th1 adjuvants through in vitro assays of their immune stimulation of spleen cells derived from naive BALB/c mice. Two of the tested six fractions (namely F2 and F4) were characterized as polysaccharide-rich fractions, and were able to induce high levels of IFN- $\gamma$, and low levels of IL-4 and IL-10 in the spleen cells. The efficacy of adjuvant action against L. infantum was evaluated in BALB/c mice, with these fractions being administered together with a recombinant antigen, LiHyp1, which was previously evaluated as a vaccine candidate, associated with saponin, against visceral leishmaniasis (VL). The associations between LiHyp1/F2 and LiHyp1/F4 were able to induce an in vivo Th1 response, which was primed by high levels of IFN- $\gamma$, IL-12, and GM-CSF, by low levels of IL- 4 and IL-10; as well as by a predominance of IgG2a antibodies in the vaccinated animals. After infection, the immune profile was maintained, and the vaccines proved to be effective against $L$. infantum. The immune stimulatory effects in the BALB/c mice proved to be similar when comparing the F2 and F4 fractions with a known Th1 adjuvant (saponin), though animals vaccinated with saponin did present a slight to moderate inflammatory edema on their hind footpads. In conclusion, the F2 and F4 fractions appear to induce a Th1-type immune response and, in this context, they could be evaluated in association with other protective antigens against Leishmania, as well as in other disease models.

(c) 2015 Elsevier Inc. All rights reserved.

\section{Introduction}

Visceral leishmaniasis (VL) is mainly caused by two species of parasites, the anthroponotic Leishmania donovani and the zoonotic L. infantum, for which a variety of canids serve as animal reservoirs (Ready, 2014). The incidence of VL has been estimated to be around 500,000 cases per year, with over 50,000 deaths worldwide. Approximately $90 \%$ of the cases are registered in Bangladesh, Brazil, Ethiopia, India, Nepal, and Sudan (Alvar et al., 2012). Additionally, in the nearly 98 countries that have reported the disease; 35 of them have already registered cases of VL and HIV co-infection (World Health Organization, 2010).

The treatment of the disease is still based on the administration of pentavalent antimonial compounds; however, several side effects registered in the patients, as well as the increased parasite resistance to the drugs, have also caused serious problems (Croft and Coombs, 2003; Minodier and Parola, 2010). Therefore, the development of new strategies to prevent leishmaniasis has become a high-priority (Costa et al., 2011; Lee et al., 2012).

The evidence of life-long immunity has inspired the development of vaccination protocols against the disease, but few have progressed beyond the experimental stage (Chávez-Fumagalli et al., 2010; Coelho et al., 2003; Costa et al., 2014; Ramírez et al., 2013, 2014). In these studies, the type- 1 cell-mediated immunity has proven to be important as a protective response against leishmaniasis. This response commonly depends on an IL-12-driven response, leading to an IFN- $\gamma$ and IL-2 production. Substantial up regulation of inducible NO synthase by IFN- $\gamma$ generates NO from splenic and liver cells, thereby controlling parasite replication in these organs (Carrión et al., 2006; Oliveira et al., 2011). In contrast, IL-4, IL-10, IL-13, and TGF- $\beta$ are major disease promoting cytokines, in turn leading to the suppression of the Th1 response (Afonso and Scott, 1993; Martins et al., 2013). Thus, vaccines capable of inducing the development of a Th1 immune response, primed by the production of cytokines, such as IFN- $\gamma$ and IL-12, and associated with low levels of IL-4 and IL-10, could be considered promising candidates to protect against the Leishmania sp. infection (Costa et al., 2014; Fernandes et al., 2012).
Adjuvants of the immune response are defined as products capable of enhancing and/or changing an antigen-specific immune response. Biotechnological advances have enabled modern vaccines to be based on the use of rationally designed recombinant antigens associated with purified compounds as adjuvants, which present a satisfactory safety profile (Reed et al., 2013). Adjuvants can be used for multiple purposes, such as to enhance the immunogenicity of weak antigens, provide antigen-dose sparing to accelerate the immune response, reduce the need for several immunizations, and/or increase the duration of protection (Petrovsky and Aguilar, 2004). These products can be classified as immune stimulatory molecules, such as toll-like receptor ligands and saponins, or delivery systems, such as aluminum salts, emulsions, and lipid nanosystems (Fox et al., 2013).

The use of carbohydrates with adjuvant action is based on their binding to innate immune receptors (Petrovsky and Cooper, 2011). A number of carbohydrate compounds extracted from plants (Rajput et al., 2007), bacteria (Koike et al., 1998), and yeast (Pillemer and Ecker, 1941) have emerged as promising vaccine adjuvant candidates. Their protective effects induced in immunotherapy have also been proven to act against L. amazonensis (Yatawara et al., 2009), L. donovani (Cook et al., 1982), and L. major (Al Tuwaijri et al., 1987). In addition, carbohydrates' adjuvant activity against L. donovani in a golden hamster model has been registered (Cook et al., 1982; Obaid et al., 1989). In parallel to the use of carbohydrates, the employ of protein-bound polysaccharides has also been evaluated in a wide range of biological applications (Maehara et al., 2012; Ooi and Liu, 2000), and recent studies have shown their use as immune adjuvants (Engel et al., 2013).

Recently, our group demonstrated the in vitro (Valadares et al., 2011) and in vivo antileishmanial activity of Agaricus blazei against L. amazonensis and L. infantum (Valadares et al., 2012a, 2012b, respectively). Compounds, such as $\beta$-glucans, tannins, glycoproteins, and polysaccharides were characterized in this mushroom, which may well be responsible for the immunological activity of the fungus (Bernardshaw et al., 2005; Gonçalves et al., 2012; Valadares et al., 2012a). Taking this into account, in the present study, six polysaccharide-rich fractions were purified from Agaricus blazei, and 
evaluated in in vitro experiments as inducers of the Th1 immune response. The adjuvant fractions that presented the best results were used in association with a recombinant antigen of L. infantum, rLiHyp1 (Martins et al., 2013); and the subsequent vaccine was evaluated as regards the protection of BALB/C mice against L. infantum, using saponin as a control.

\section{Materials and methods}

\subsection{Ethics statement}

Experiments were performed in compliance with the National Guidelines, as set forth by the Institutional Animal Care (Law number 11.794, 2008), and the Committee on the Ethical Handling of Research Animals from the Federal University of Minas Gerais (UFMG), who approved this study under protocol number 067/2013.

\subsection{Mice and parasites}

Female BALB/c mice ( 8 weeks of age) were obtained from the breeding facilities of the Department of Biochemistry and Immunology, Institute of Biological Sciences (ICB), UFMG; and were maintained under specific pathogen-free conditions. Experiments were carried out using the L. infantum (MOM/BR/1970/BH46) strain. Parasites were grown at $24{ }^{\circ} \mathrm{C}$ in Schneider's medium (Sigma-Aldrich, St. Louis, MO, USA), supplemented with $10 \%$ heatinactivated fetal bovine serum (FBS; Sigma-Aldrich), $20 \mathrm{mM}$ L-glutamine, $200 \mathrm{U} / \mathrm{mL}$ penicillin, and $100 \mu \mathrm{g} / \mathrm{mL}$ streptomycin, at $\mathrm{pH}$ 7.4. The soluble Leishmania antigenic (SLA) extract was prepared from $1 \times 10^{9}$ stationary-phase promastigotes, as described (Coelho et al., 2003).

\subsection{Purification of polysaccharide-rich fractions from Agaricus blazei}

The technical protocol used to obtain the polysaccharide-rich fractions from Agaricus blazei was adapted from Camelini et al. (2005) and Meng et al. (2008). Briefly, $20 \mathrm{~g}$ of fresh and dried fruiting body of Agaricus blazei (Energy Vita ${ }^{\circledR}$, lot \#1, harvest in 2013) was macerated in $400 \mathrm{~mL}$ of ethanol $85 \%(\mathrm{v} / \mathrm{v})$, with the solution heated for $3 \mathrm{~h}$ at $80^{\circ} \mathrm{C}$. Next, the solution was filtered in a vacuum filtration system using coarse filter paper, and the supernatant was discarded. The pellet was dissolved in $350 \mathrm{~mL}$ of distilled water, heated at $100{ }^{\circ} \mathrm{C}$ for $3 \mathrm{~h}$, and then filtered with a vacuum filtration system. Afterwards, 4 vol of ethanol PA (v/v) was added. The solution was heated to above $80{ }^{\circ} \mathrm{C}$ to reduce the final volume, and the remaining solution was dialyzed with distilled water for 4 days, using membranes with the pore size of 40,000 Da. Afterwards, the solution was lyophilized to obtain the total extract. Approximately $500 \mathrm{mg}$ of the extract was dissolved in $50 \mathrm{~mL}$ of deionized water, and the material was centrifuged at $12,500 \times \mathrm{g}$ for $30 \mathrm{~min}$ at $4{ }^{\circ} \mathrm{C}$. The supernatant was collected and further centrifuged at $37,500 \times \mathrm{g}$ for $40 \mathrm{~min}$, and then passed through a $0.45 \mu \mathrm{M}$ filter (Millipore ${ }^{\circledR}$, Merck, Billerica, MA, USA). The collected supernatant was placed in a DEAE-Sepharose ion exchange column, at which time six fractions were obtained and their carbohydrate content was determined using a phenol-sulphuric acid spectrophotometric method (Dubois et al., 1956); the protein content was determined using the Lowry method (Lowry et al., 1951); and the phenol compound content was determined following the Ainsworth method (Ainsworth and Gillespie, 2007). The technical procedure was repeated three times, and the purified fractions were stored at $-80^{\circ} \mathrm{C}$ until use.

\subsection{Structural characterization of the best adjuvant fractions from Agaricus blazei}

The characterization of the fractions that presented the best in vitro immune stimulation results was performed by FTIR spectroscopy, and analyzed by transmittance IR spectroscopy, in the form of $\mathrm{KBr}$ discs, using a Perkin Elmer Spectrum 1000 FTIR spectrometer. ${ }^{1} \mathrm{H}$ and ${ }^{13} \mathrm{C}$ NMR spectra of solutions in D2O were recorded at room temperature using a Fourier Transform Bruker Avance DRX 500 spectrometer, with an inverse multinuclear gradient probehead equipped with z-shielded gradient coils, together with Silicon Graphics. The sodium 2,2-dimethylsilapentane-5-sulphonate (DSS) was used as the internal standard for the experiments $(0.00 \mathrm{ppm}$ for $\left.{ }^{1} \mathrm{H}\right)$.

\subsection{Evaluation of the in vitro immune stimulation}

Spleen cells obtained from BALB/c mice $(n=8)$ were collected, pooled and in vitro cultured in 24-well plates (Nunc, Nunclon ${ }^{\circledR}$, Roskilde, Denmark), at $5 \times 10^{6}$ cells per $\mathrm{mL}$, in duplicate. As a negative control, cells were incubated without any stimulus in complete RPMI 1640 medium (Sigma-Aldrich), which was composed by medium plus $10 \%$ FBS, 20 mM L-glutamine, $200 \mathrm{U} / \mathrm{mL}$ penicillin, and $100 \mu \mathrm{g} / \mathrm{mL}$ streptomycin, at $\mathrm{pH}$ 7.4. The purpose was to evaluate the cells' natural stimulation by technical manipulation. In parallel, cells were separately stimulated with each one of the six individual fractions (100 $\mu \mathrm{g}$ per well, each one), or saponin (250 $\mu \mathrm{g}$ per well), for $48 \mathrm{~h}$ at $37{ }^{\circ} \mathrm{C}$ in $5 \% \mathrm{CO}_{2}$. Cells were also stimulated with concanavalin $\mathrm{A}$ (ConA; $5 \mu \mathrm{g}$ per well), as a positive control (data not shown). The IFN- $\gamma$, IL- 4 and IL-10 levels were determined in the culture supernatants, using commercial kits (BD OptEIA, Pharmingen, San Diego, CA, USA), according to manufacturer's instructions.

\subsection{Immunization and challenge infection}

After the evaluation of the in vitro immune stimulation results, two (F2 and F4) of the six fractions were selected, based on their capacity to induce a polarized Th1 immune response, and were evaluated in association with the LiHyp1 protein. The recombinant (rLiHyp1) protein was purified as described (Martins et al., 2013 ). For the vaccination, BALB/c mice ( $n=8$, per group) were immunized subcutaneously in their left hind footpad with $25 \mu \mathrm{g}$ of rLiHyp1 associated with $25 \mu \mathrm{g}$ of saponin (Quillaja saponaria bark saponin, Sigma); or associated with $100 \mu \mathrm{g}$ of F2 or F4. Other animals received only adjuvants, the rLiHyp1 or diluent (saline). Three doses were administered at 2-week intervals. Four weeks after the last immunization, animals ( $n=4$, per group) were euthanized for the analysis of the immune response elicited by vaccination. At the same time, the remaining animals ( $n=4$, per group) were infected subcutaneously in the right hind footpad with $1 \times 10^{7}$ stationaryphase promastigotes of $L$. infantum. After 10 weeks, the animals were euthanized, and the liver, spleen, bone marrow (BM), and paws' draining lymph nodes (dLN) were collected to analyze the parasite burden and evaluate the immune response. The vaccination experiments were repeated twice, and presented similar results.

\subsection{Estimation of parasite load}

The liver, spleen, BM and dLN were collected from the euthanized animals for parasite quantification, following a limitingdilution technique (Coelho et al., 2003). Briefly, organs were weighed and homogenized using a glass tissue grinder in sterile PBS. Tissue debris were removed by centrifugation at $150 \times g$, and cells were concentrated by centrifugation at $2000 \times$ g. Pellets were resuspended in $1 \mathrm{~mL}$ of Schneider's insect medium supplemented with $20 \%$ FBS. Two hundred and twenty microliters was plated onto 
96-well flat-bottom microtiter plates (Nunc), and diluted in logfold serial dilutions using supplemented Schneider's medium, to a $10^{-1}-10^{-12}$ dilution. Each sample was plated in triplicate and read 7 days after the beginning of the culture, at $24^{\circ} \mathrm{C}$. Pipette tips were discarded after each dilution to avoid carrying adhered parasites from one well to another. Results are expressed as the negative log of the titer (i.e., the dilution corresponding to the last positive well) adjusted per microgram of tissue.

\subsection{Cytokine production}

Splenocyte cultures and cytokine assays were performed before infection, and at 10th week after challenge, as described (Coelho et al., 2003). Briefly, single-cell preparations from spleen tissue were plated in duplicate in 24-well plates (Nunc) at $5 \times 10^{6}$ cells $/ \mathrm{mL}$. Cells were incubated in complete RPMI 1640 medium (non-stimulated, negative control), or separately stimulated with SLA L. infantum $\left(25 \mu \mathrm{g} \mathrm{mL} \mathrm{m}^{-1}\right)$ or rLiHyp $1\left(20 \mu \mathrm{g} \mathrm{mL}^{-1}\right)$, at $37^{\circ} \mathrm{C}$ in $5 \% \mathrm{CO}_{2}$ for $48 \mathrm{~h}$. IFN- $\gamma$, IL-4, IL-10, IL-12, and GM-CSF levels were assessed in the supernatants by a sandwich ELISA provided in commercial kits (BD OptEIA TM set mouse IFN- $\gamma$, IL-4, IL-10, IL-12 and GM-CSF; Pharmingen, USA); following manufacturer's instructions. In order to block IL$12, \mathrm{CD}^{+}$, and $\mathrm{CD}^{+}$mediated $\mathrm{T}$ cell cytokine release, spleen cells of mice vaccinated and challenged were in vitro stimulated with SLA L. infantum $\left(25 \mu \mathrm{g} \mathrm{mL}^{-1}\right)$, and incubated in the presence of $5 \mu \mathrm{g}$ per $\mathrm{mL}$ of monoclonal antibodies ( $\mathrm{mAb}$ ) against mouse IL-12 (C17.8), $\mathrm{CD}^{+}$(GK 1.5), or $\mathrm{CD}^{+}$(53-6.7). Appropriate isotypes-matched controls - rat IgG2a (R35-95) and rat IgG2b (95-1) - were employed in the assays. Antibodies (no azide/low Endotoxin ${ }^{\mathrm{TM}}$ ) were purchased from BD (Pharmingen, USA).

\subsection{Analysis of the humoral response}

The rLiHyp1- or SLA L. infantum-specific IgG1 and IgG2a antibodies were measured by ELISA, as described (Coelho et al., 2003). Previous titration curves were performed to determine the most appropriate antigen concentration, and antibody dilution to be used. Briefly, Falcon flexible microtiter immunoassay plates (Becton Dickinson) were coated with SLA L. infantum (1.0 $\mu$ g per well) or rLiHyp1 $(1.0 \mu$ g per well), diluted in $100 \mu \mathrm{L}$ coating buffer $(50 \mathrm{mM}$ carbonate buffer) $\mathrm{pH} 9.6$, for $18 \mathrm{~h}$ at $4{ }^{\circ} \mathrm{C}$. After this, free binding sites were blocked using $200 \mu \mathrm{L}$ of PBS-T (phosphate buffer saline $1 \times$ plus Tween $200.05 \%$ ) buffer containing $5 \%$ casein for $1 \mathrm{~h}$, at $37^{\circ} \mathrm{C}$. After washing the plates 5 times using PBS-T, they were incubated with $100 \mu \mathrm{L}$ of individual mice sera for $1 \mathrm{~h}$ at $37^{\circ} \mathrm{C}$. Samples were diluted 1:50 in PBS-T containing 0.5\% casein solution. Plates were washed 5 times using PBS-T, and incubated with the peroxidase-labeled antibodies specific to mouse IgG1 or IgG2a isotypes (Sigma Aldrich) diluted at 1:5000, and incubated for $1 \mathrm{~h}$ at $37^{\circ} \mathrm{C}$. After washing 5 times with PBS-T, the reaction was developed through incubation with $\mathrm{H}_{2} \mathrm{O}_{2}$, ortho-phenylenediamine and citrate-phosphate buffer $\mathrm{pH} 5.0$, for $30 \mathrm{~min}$ in the dark. The reaction was stopped by adding $25 \mu \mathrm{L} \mathrm{H}_{2} \mathrm{SO}_{4} 2 \mathrm{~N}$, and optical density was read in an ELISA microplate spectrophotometer (Molecular Devices, Spectra Max Plus, Canada), at $492 \mathrm{~nm}$.

\subsection{Statistical analysis}

Statistical analysis was performed using the GraphPad Prism software (version 6.0 for Windows). Results were expressed by mean \pm standard deviation (SD) of the groups. Outliers were evaluated using the ROUT test and excluded from statistical analyses. The normality analysis of the data was performed using the D'Agostino and Pearson test. Statistical analysis of the results of immunized and/ or infected mice was performed by one-way analysis of variance (ANOVA), using Tukey's post-test for multiple comparisons between the groups. Differences were considered significant when $P<0.05$. Data presented in this study are representative of two independent vaccination experiments, which presented similar results.

\section{Results}

\subsection{Chemical characterization of the purified fractions from}

Agaricus blazei and the in vitro selection of best adjuvant fractions

To select the polysaccharide-rich fractions derived from Agaricus blazei in order to perform the in vitro immune stimulation experiments, the polysaccharide, protein, and phenolic compound contents were determined, and the results are showed in Fig. 1. The total extract of Agaricus blazei (ABP) was used as a positive control for the presence of these compounds. In evaluating the protein content, only the F5 fraction presented high levels, whereas the other fractions did not present significant levels (Fig. 1A). In the evaluation of the presence of carbohydrates, the F2, F3, and F4 fractions presented the highest levels of its content, and were therefore selected (Fig. 1B); however, upon evaluating the content of phenolic compounds (Fig. 1C), it could be observed that the F3 fraction presented the highest levels, and for this reason it was not selected for the experiments. Fig. 1D shows the ratio between the carbohydrate and protein levels of all of the fractions. Evaluating the results, the F2 and F4 fractions were selected, as they presented the best results of polysaccharide content, and were employed in the in vitro experiments of immune stimulation.

To evaluate the in vitro immune stimulation of the two polysaccharide-rich fractions obtained earlier, spleen cells taken from naive BALB/c mice were collected, pooled, and separately stimulated with each of the two purified fractions (F2 and F4), at which time the IFN- $\gamma$, IL-4, and IL-10 levels were determined in the supernatant cultures (Fig. 1E). Non-stimulated cells (only incubated in medium) were used as a negative control, while the spleen cells stimulated with saponin were used as a positive control. From the results, it was possible to observe that both the F2 and F4 fractions were able to stimulate a high production of IFN- $\gamma$, which was associated with low levels of IL-4 and IL-10. The results of these two fractions were similar to those found with the saponin stimulus (Martins et al., 2013) and, in this context, these two fractions were selected and evaluated as Th1 response adjuvants, in association with the rLiHyp1 protein, as a vaccine against L. infantum.

\subsection{Structural characterization of adjuvant fractions using ${ }^{1} \mathrm{H} N M R$ and ${ }^{13} \mathrm{C}$ NMR spectroscopy}

In an attempt to characterize the selected fractions structurally, FTIR spectra of the fractions were taken, and the results are presented as a Supplementary figure (Fig. S1). Although the F2 and F4 fractions had been characterized as rich-polysaccharide fractions, they present some differences in their chemical contents. Thus, F2 fraction possesses a higher degree of carbohydrates and phenolic compounds than the F4 fraction. This last fraction presents a higher degree of proteins, in comparison to F2 fraction. Like a representative analysis, data derived from the F4 fraction were showed (Fig. S1). In their analysis, the main attributions of the absorption peaks reveal characteristic glycosidic structures: $\mathrm{OH}$ stretching (3000-3500 $\left.\mathrm{cm}^{-1}\right)$, CO stretching $\left(1022 \mathrm{~cm}^{-1}\right), \mathrm{C}-\mathrm{O}-\mathrm{C}$ stretching $\left(1162 \mathrm{~cm}^{-1}\right)$, and anomeric $C_{1} \mathrm{H}$ group vibration $\left(1082 \mathrm{~cm}^{-1}\right)$. The protein attributions were observed with characteristic absorption peaks at 1649, 1413 (characteristic absorption bands related to carboxylate anion), and $1242 \mathrm{~cm}^{-1}$. These attributions suggest the presence of a protein-glucans complex (Fig. S1A). The ${ }^{1} \mathrm{H}$ NMR spectrum obtained from the F4 fraction is also shown (Fig. S1B). The anomeric region and the other carbon protons in beta-configuration were observed. The ${ }^{1} \mathrm{H}$ chemical shifts in the anomeric region are 

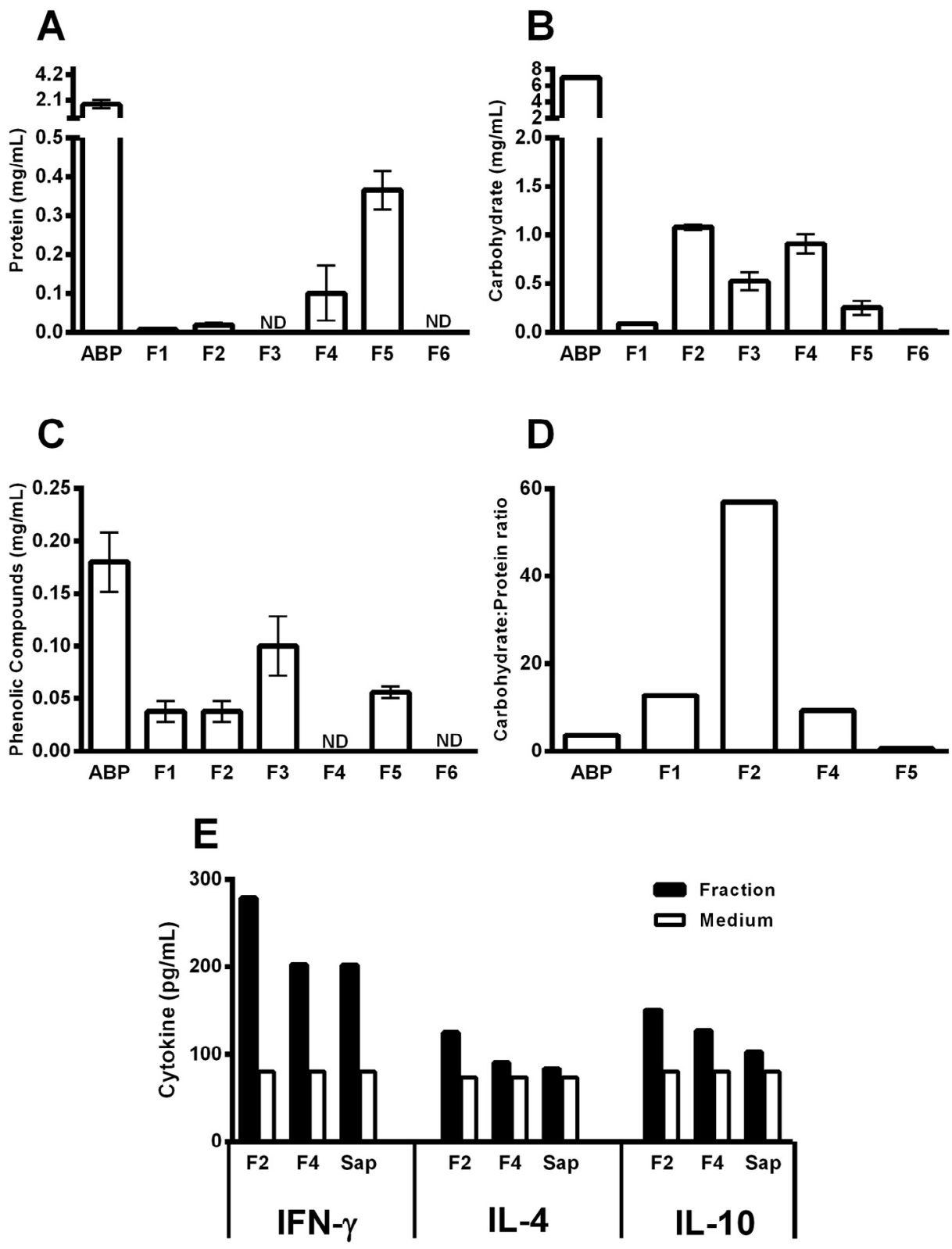

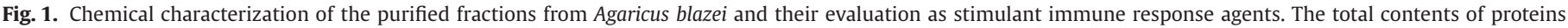

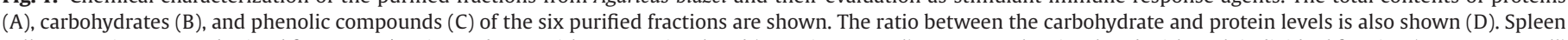

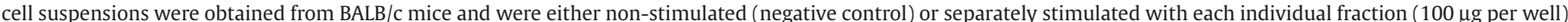

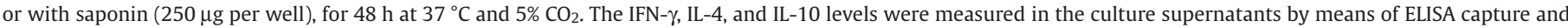

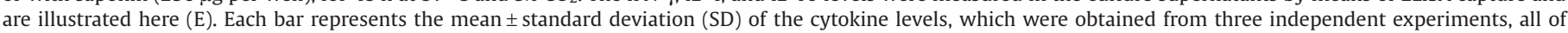
which presented similar results.

observed between 4 and 6 ppm. Signals at 4.1 and $4.5 \mathrm{ppm}$ were observed corresponding to the beta-configuration and in the region between 4.9 and $5.6 \mathrm{ppm}$, corresponding to alpha-configuration. The chemical shifts from 3.3 to $4.0 \mathrm{ppm}$ were assigned to the protons of carbons $\mathrm{C} 2-\mathrm{C} 6$ of the glycosidic ring. The attributions observed at $0.5-3.0 \mathrm{ppm}$ were considered to be from proteins, given that the $\mathrm{N}-\mathrm{CH}_{3}$ and $\mathrm{N}-\mathrm{H}$ groups are generally found within this range. The ${ }^{13} \mathrm{C}$ NMR spectrum of the F4 fraction is also shown here (Fig. S1C). The assignments of the representative peaks of carbohydrates included: anomeric carbon (C1) signals of glycosides assigned to approximately $105 \mathrm{ppm}$, and C2-C6 from the glycosidic ring assigned to $70-80 \mathrm{ppm}$. The intensity of signals reveals that the polysacarides of the fractions are constituted by $\beta$-glucans.

\subsection{Immunogenicity of rLiHyp1 protein associated or not with saponin, F2 or F4 fractions}

The immunogenicity of rLiHyp1 protein associated with the F2 or F4 fractions, or saponin, as well as administered alone, was evaluated in BALB/c mice; 4 weeks after the last vaccine dose, and before the challenge infection (Fig. 2). Spleen cells from mice vaccinated with rLiHyp1/F2, rLiHyp1/F4, or rLiHyp1/saponin produced significantly higher levels of IFN- $\gamma$, IL-12, and GM-CSF than did those secreted by spleen cells from all control groups (saline, F2, F4, saponin, or rLiHyp1 groups), following in vitro stimulation with recombinant protein. In this context, rLiHyp1/F2, rLiHyp1/F4, or rLiHyp1/saponin groups produced significantly higher levels of these 


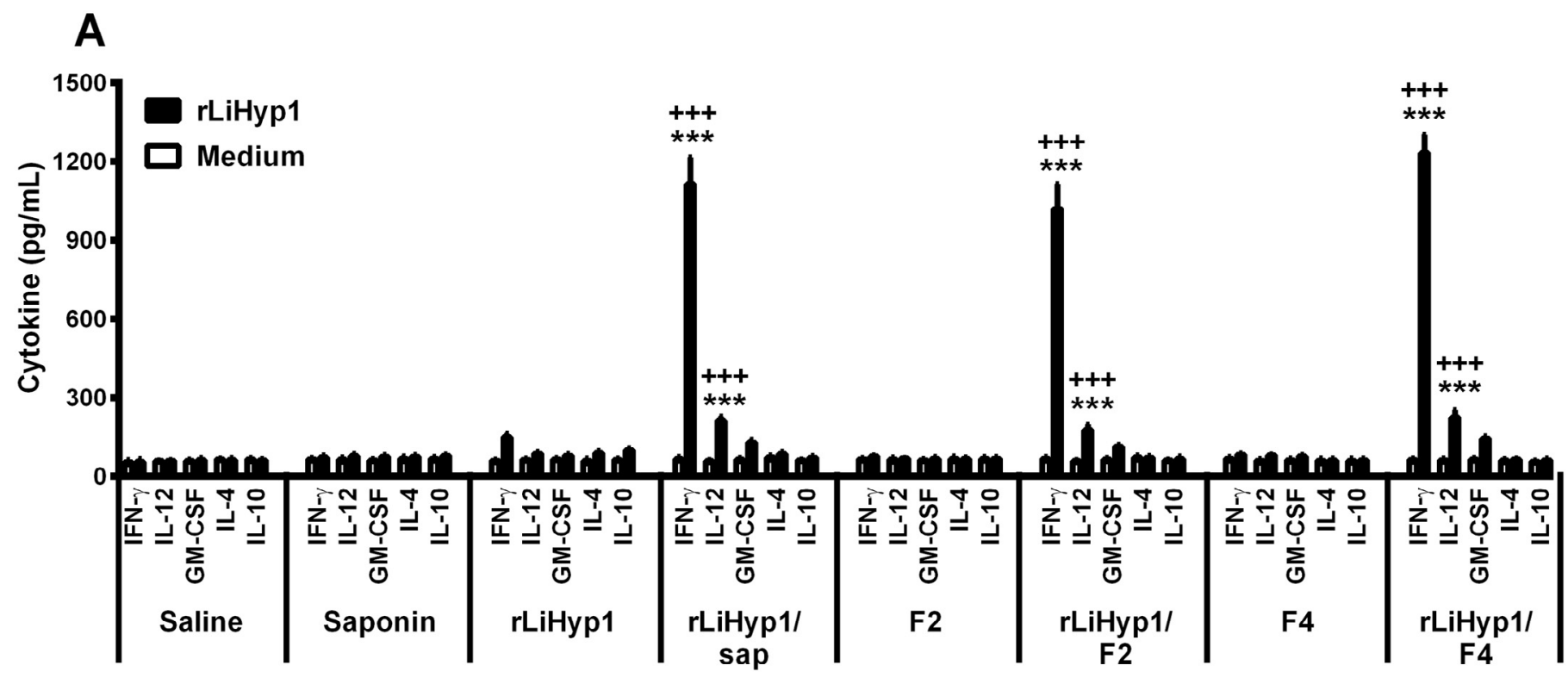

B

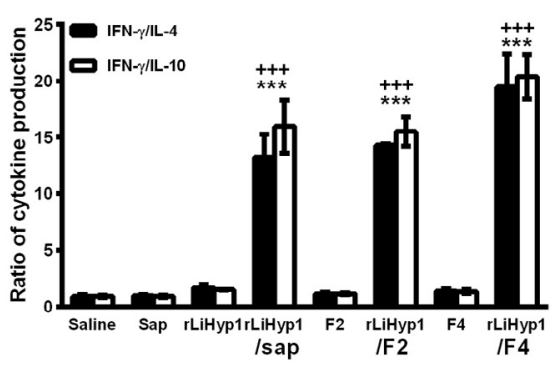

C

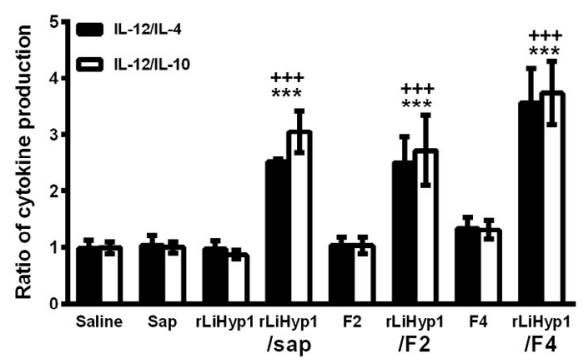

D

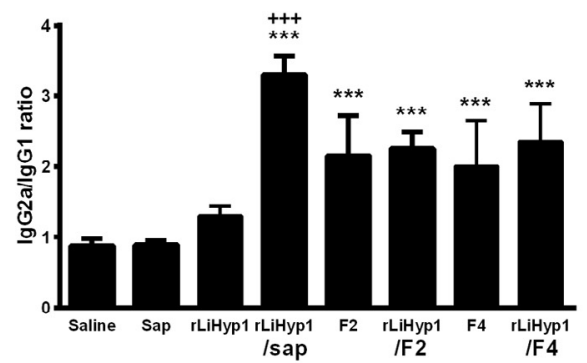

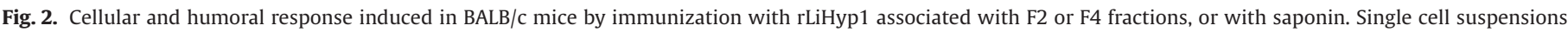

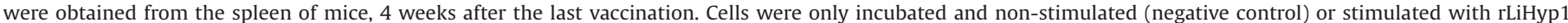

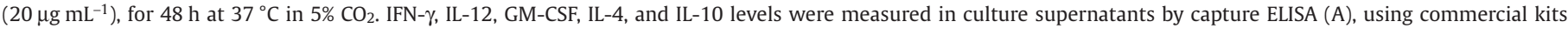

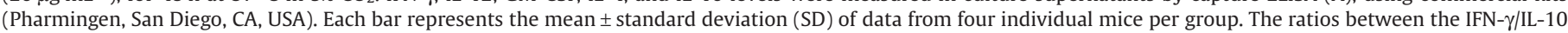

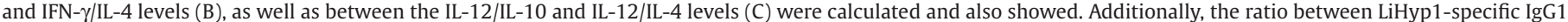

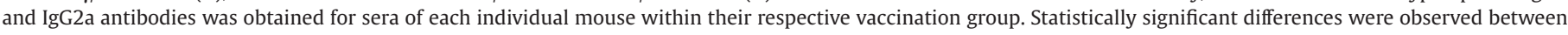
the rLiHyp1/F2, rLiHyp1/F4 or rLiHyp1/saponin groups, when compared to saline $\left({ }^{* * *} P<0.0001\right)$ and rLiHyp1 $\left({ }^{++} P<0.0001\right)$ groups $(\mathrm{D})$.

cytokines in comparison to the saline group $\left({ }^{* * *} \mathrm{P}<0.0001\right)$, as an infection control; as well as in relation to the rLiHyp1 group, as an adjuvant' efficacy control $\left({ }^{++} P<0.0001\right)$. In addition, no significant difference in the IL- 4 and IL-10 production could be observed in any group, after stimulation with the rLiHyp1 protein (Fig. 2A). The ratio between IFN- $\gamma /$ IL- 4 and IFN- $\gamma /$ IL-10 levels, as well as between IL-12/IL-4 and IL-12/IL-10 levels, showed that vaccinated animals with rLiHyp1/F2, rLiHyp1/F4, or rLiHyp1/saponin presented a predominant Th1 response after LiHyp1-stimulus (Fig. 2B and $C$, respectively). Moreover, in these vaccinated animals, the rLiHyp1-specific antibody production was predominantly found in the IgG2a isotype (Fig. 2D). No significant difference in the cytokine or antibody production could be observed between the rLiHyp1/F2, rLiHyp1/F4, and rLiHyp1/saponin groups.

\subsection{Protective efficacy against L. infantum}

Evaluating if the immunization with rLiHyp1 protein associated with F2, F4, or saponin adjuvants was able to induce a Th1 response in the vaccinated animals and protect against challenge infection, this study investigated whether or not the vaccination would, in fact, protect against L. infantum. For this, after the three vaccine doses, an infection was performed and the animals were followed-up over a 10-week period, at which time the parasite burden was evaluated and expressed as the negative log of the titer in the liver, spleen, dLN, and BM of the vaccinated and infected animals (Fig. 3). In the results, reductions in the number of parasites were observed in all of the evaluated organs in the vaccinated mice with rLiHyp1/F2, rLiHyp1/F4, or rLiHyp1/saponin, when compared to the control groups (saline, F2, F4, saponin, and rLiHyp1 groups). In this context, vaccinated mice with rLiHyp1/F2, when compared to the saline and F2 groups, presented reductions in the parasite load in the liver (3.2 and 2.7-log reductions, respectively; Fig. 3A), spleen (2.2 and 2.0-log reductions, respectively; Fig. 3B), dLN (2.6 and 2.1-log reductions, respectively; Fig. 3C), and BM (2.5 and 2.3-log reductions, respectively; Fig. 3D). In addition, vaccinated mice with rLiHyp1/F4, when compared to saline and F4 groups, presented reductions in the parasite load in the liver (4.0 and 3.1log reductions, respectively; Fig. 3A), spleen (3.1 and 2.8-log reductions, respectively; Fig. 3B), dLN (4.1 and 3.3-log reductions, respectively; Fig. 3C), and BM (4.2 and 3.0-log reductions, respectively; Fig. 3D). In the same manner, immunized mice with rLiHyp1/saponin, when compared to the saline and rLiHyp1 groups, presented reductions in the parasite load in the liver (3.8 and 


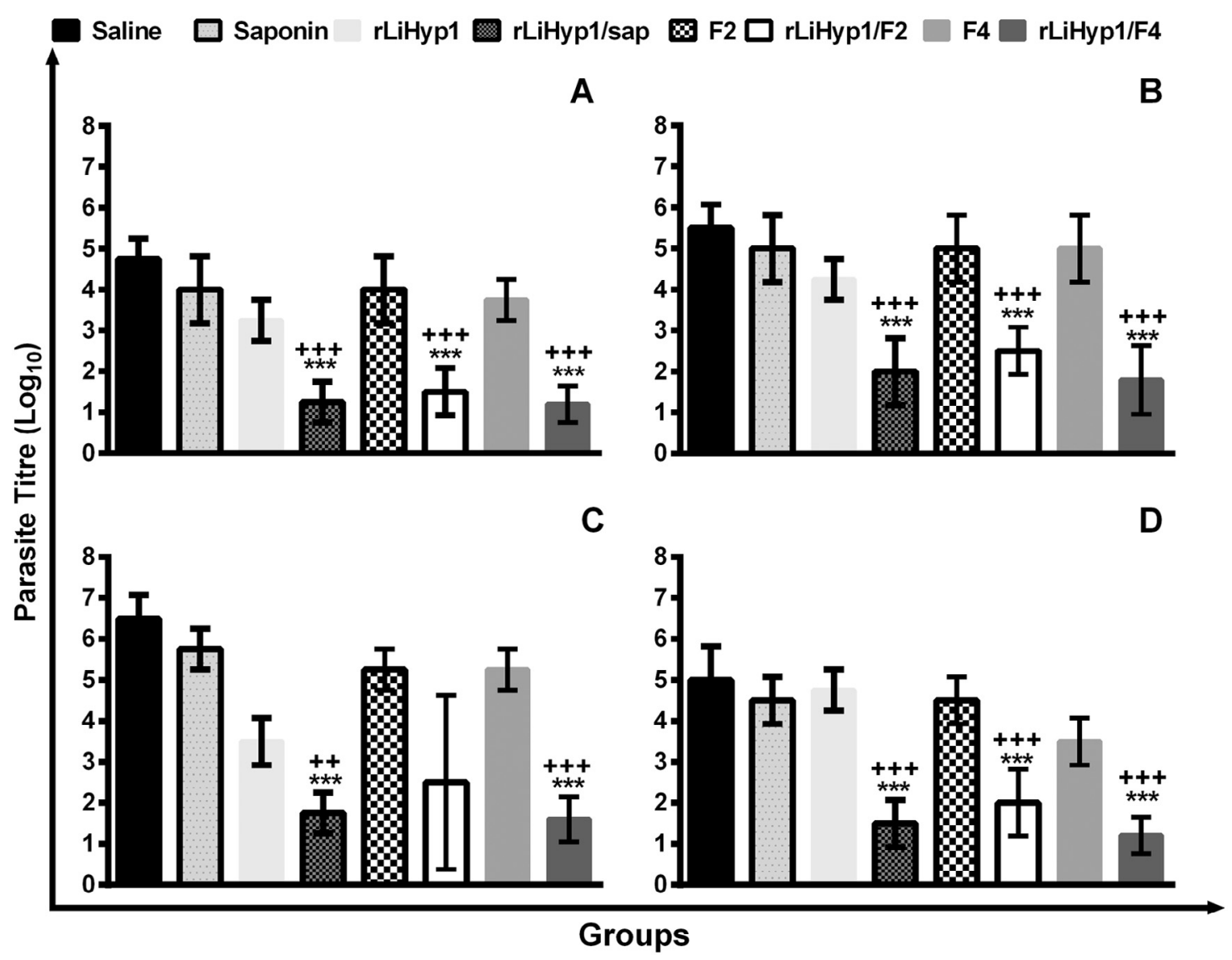

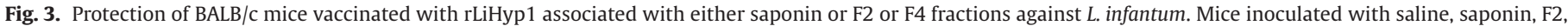

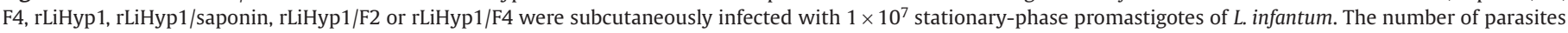

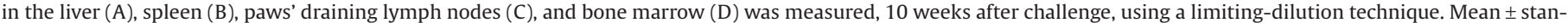

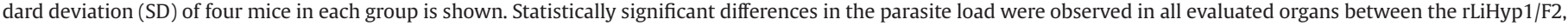

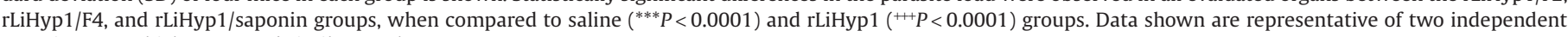
experiments, which presented similar results.

2.6- $\log$ reductions, respectively), spleen (2.8 and 2.1-log reductions, respectively), dLN (3.7 and 2.7-log reductions, respectively), and BM (3.3 and 2.3-log reductions, respectively).

In all cases, the results showed the protection induced by rLiHyp1 protein against L. infantum, when associated with a Th1 adjuvant, as previously described (Martins et al., 2013). No difference could be observed in the parasite burden between the rLiHyp1/F2, rLiHyp1/F4, and rLiHyp1/saponin groups. In addition, the immunization with rLiHyp1, without adjuvant, was unable to induce protection against $L$. infantum, demonstrating the necessity of the association of immune adjuvants to vaccine candidates against leishmaniasis.

\subsection{Cellular and humoral responses elicited after infection}

The cytokine production in the supernatants of spleen cell cultures stimulated with SLA was analyzed 10 weeks after infection (Fig. 4). Spleen cells derived from mice vaccinated with rLiHyp1/F2, rLiHyp1/F4, or rLiHyp1/saponin produced significantly higher levels of SLA-specific IFN- $\gamma$, IL-12, and GM-CSF cytokines, than did those secreted by spleen cells from control groups (saline, F2, F4, saponin, and rLiHyp1 groups; Fig. 4A). By contrast, the SLA-driven production of IL-4 and IL-10 showed that vaccination with the rLiHyp1 protein associated with F2 or F4 fractions, or saponin, induced very low levels of these cytokines in the vaccinated and infected animals. The ratio between IFN- $\gamma /$ IL- 4 and IFN- $\gamma /$ IL-10 levels (Fig. 4B), as well as between the IL-12/IL-4 and IL-12/IL-10 levels (Fig. 4C), indicated that these animals had developed a Th1 response. Using the rLiHyp1 protein as a stimulus, the Th1 profile was also maintained after the infection (data not shown). In the antibody production, it was possible to observe that vaccinated mice with rLiHyp1/F2, rLiHyp1/F4, or rLiHyp1/saponin, and infected mice presented a predominance of SLA-specific IgG2a isotypes, which was significantly higher than the IgG1 levels (Fig. 4D). This profile could also be observed when using rLiHyp1 as an antigen in the experiments (data not shown).

The present study evaluated the contribution of $\mathrm{CD} 4^{+}$and $\mathrm{CD} 8^{+}$ $\mathrm{T}$ cells, as well as the dependence of IL-12 production for the SLAspecific IFN- $\gamma$ production from the spleen cells of mice immunized with rLiHyp1/F2, rLiHyp1/F4, or rLiHyp1/saponin and infected with L. infantum (Fig. 5). The IFN- $\gamma$ production was significantly suppressed using anti-CD4 or anti-IL-12 monoclonal antibodies in the spleen cell cultures derived from mice vaccinated with rLiHyp1/ saponin (Fig. 5A), rLiHyp1/F2 (Fig. 5B), or rLiHyp1/F4 (Fig. 5C). Evaluating the results, it could be observed that the addition of antiCD8 antibodies in the cultures decreased the IFN- $\gamma$ production, as compared to the control cell cultures without treatment; however, the obtained levels of this cytokine were higher in relation to the values encountered after the use of anti-CD4 or anti-IL-12 antibodies in both vaccinated groups. These results suggest that $\mathrm{CD}^{+}$ cells were not directly involved in the IFN-gamma secretion in the present study. 

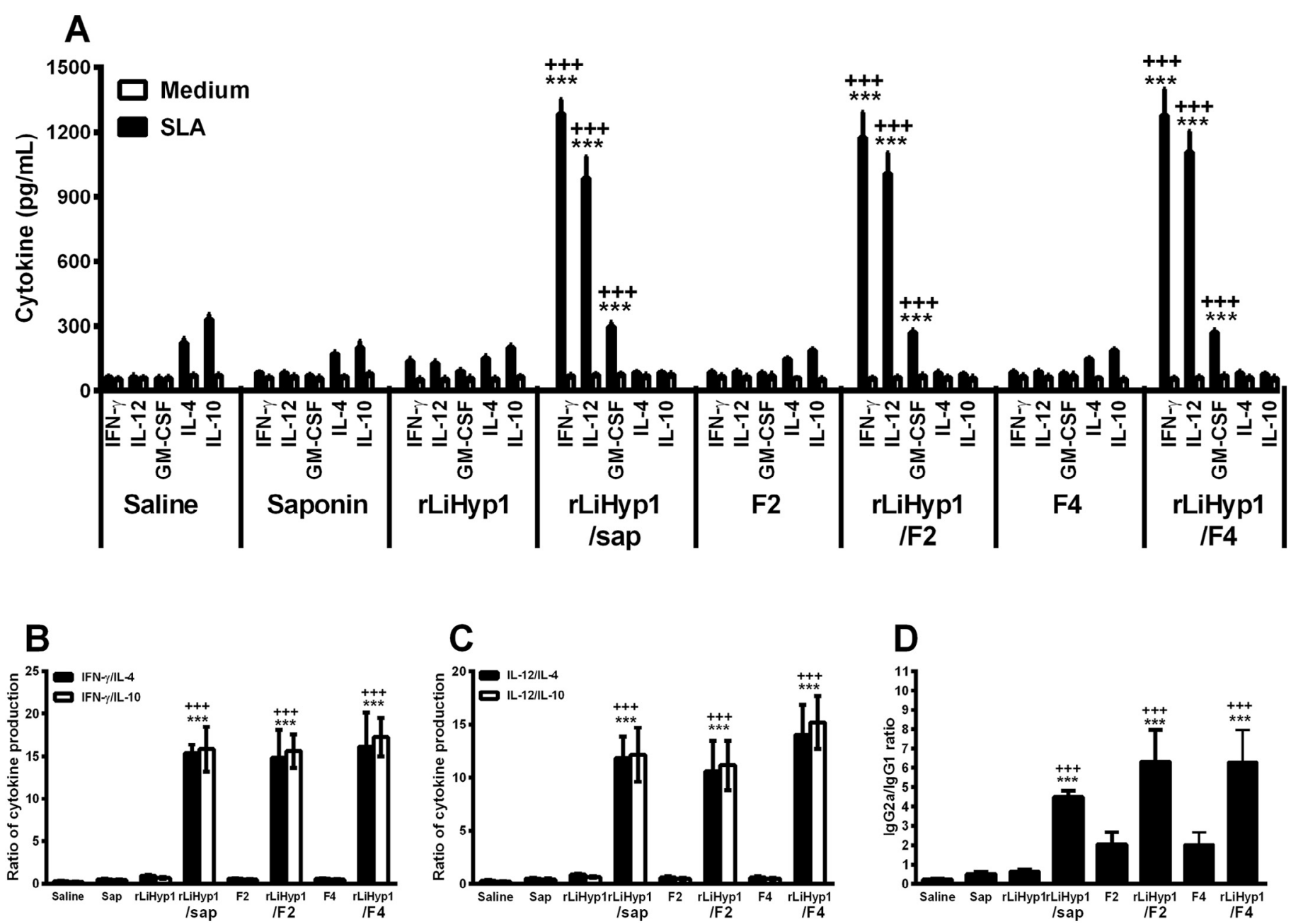

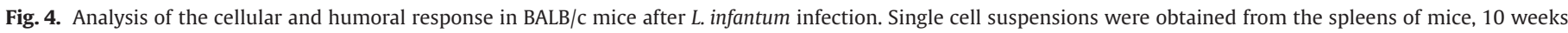

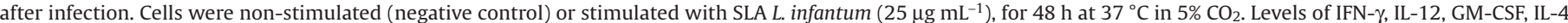

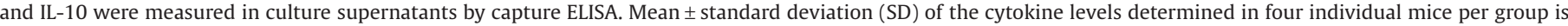

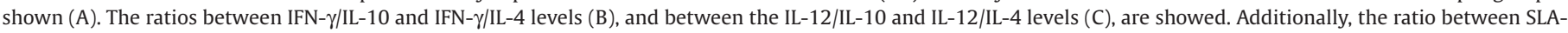

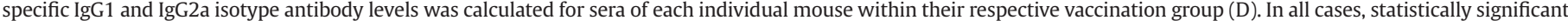
differences were observed between the rLiHyp1/F2, rLiHyp1/F4 or rLiHyp1/saponin groups, when compared to saline $\left.{ }^{* * *} P<0.0001\right)$ and ${ }^{*} L i H y p 1\left({ }^{++} P<0.0001\right)$ groups.

\section{Discussion}

Visceral leishmaniasis is one of the main neglected tropical parasite diseases, presenting a high mortality, ranking only behind malaria in the number of victims (Alvar et al., 2012). As the protection against re-infection with L. major in murine models is possible, efforts have been performed to develop a protective vaccine; however, to date, no effective vaccine has been created (Fernandes et al., 2012). An important advantage in using vaccines based on attenuated pathogens, inactivated viruses, and/or bacteria is based on the fact that they are sufficiently immunogenic, without the need to associate additional adjuvants (Coler et al., 2007; Costa et al., 2011). However, the use of these candidates would present disadvantages, mainly due to the difficulty in standardization and largescale production (Handman, 2001). By contrast, protein-based vaccines, although offering considerable advantages in terms of safety and production costs, are less immunogenic and must be supplemented with immune response adjuvants, in order to boost their immunogenicity (Cerpa-Cruz et al., 2013).

In this context, for the development of a successful vaccine, the association of a good adjuvant is desirable. Some studies have evaluated products containing this immune activity, such as interleukin-12
(Coelho et al., 2003), granulocyte macrophage-colony stimulating factor (Badaró et al., 1994), bacille Calmette-Guérin (Misra et al., 2001), montanide ISA 720 (Tonui et al., 2004), aluminum salts (Misra et al., 2001), monophosphoryl lipid A (Coler et al., 2007), CpG oligodeoxynucleotides (Stacey and Blackwell, 1999), liposomes (Kahl et al., 1989), glucans (Holbrook et al., 1981), Corynebacterium parvum (Carvalho et al., 2007), saponins (Giunchetti et al., 2007), and Freund's adjuvant (Panaro et al., 2001).

Adjuvants classified as immune stimulatory molecules present their mechanism of action based on the activation of innate immunity receptors expressed in antigen presenting cells (APCs), such as macrophages and dendritic cells, which take up and present the antigens to Thelper cells. Such receptors are used by APCs to detect pathogen-associated molecular patterns (PAMPs), called pathogen recognition receptors (PRRs) (O'Hagan and de Gregorio, 2009). Likewise, a previous study showed that fruiting bodies of Agaricus blazei present immune stimulatory activity in mice by activating $\mathrm{CD} 4^{+}$ T cells, natural killer (NK) cells, and phagocytes, as well as by increasing the production of pro-inflammatory cytokines, such as IFN- $\gamma$ and TNF- $\alpha$, using these receptors (Gonçalves et al., 2012). The authors observed that spleen cells of animals exhibited a higher radioactivity of ${ }^{99 m}$ Tecnecium-labeled leukocytes, which was associated with 


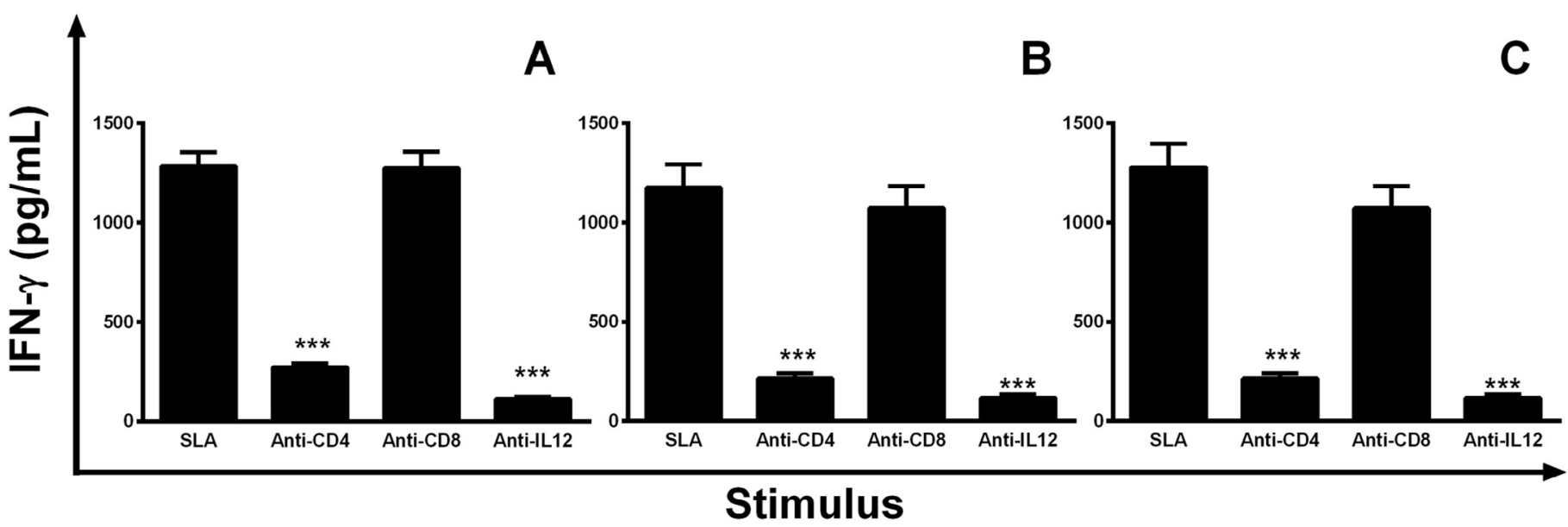

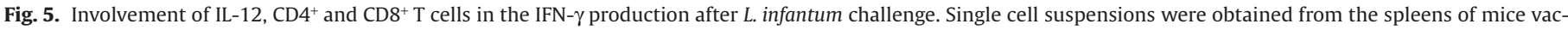

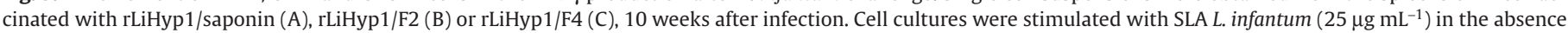

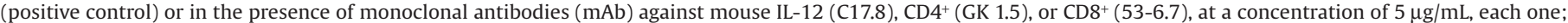

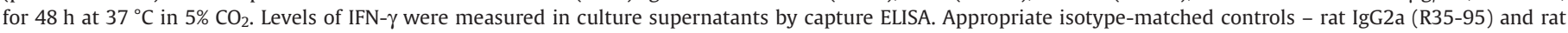

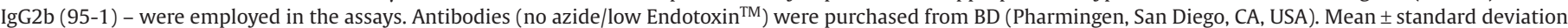

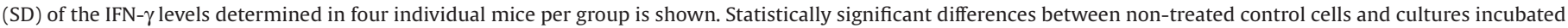
with anti-CD4, anti-CD8 or anti-IL-12 monoclonal antibodies were observed $\left({ }^{* * *} P<0.0001\right)$.

an increased number of $\mathrm{CD} 4^{+} \mathrm{T}$ cells in the spleen of the animals. In addition, the frequency of activated monocytes and neutrophils was increased in the blood of the Agaricus blazei-treated animals (Gonçalves et al., 2012).

More recently, one Agaricus blazei extract proved to be useful in enhancing the immune response to DNA vaccines against some diseases, such as hepatitis B (Wang et al., 2010), and foot-and-mouth disease (Chen and Shao, 2006); while a polysaccharide-rich fraction derived from this mushroom presented a Th1 adjuvant activity (Cui et al., 2013). In the present study, a technical protocol was performed using an Agaricus blazei total extract, from which six fractions were obtained. These products were evaluated in relation to the presence of proteins, polysaccharides, and phenolic compounds; and two of which were characterized as polysaccharide-rich fractions. Based on data described in the literature, these two fractions were used for the in vitro immune stimulation of spleen cells from naive BALB/c mice, and both were able to induce a specific and selectively higher production of IFN- $\gamma$, which was associated with low levels of IL-4 and IL-10; and the results were similar to those obtained using saponin.

Studies employing the mouse model have led to the characterization of the immune mechanisms necessary to develop organ specific immune responses, which would clear the parasites from the liver, but not from the spleen (Carrión et al., 2006). BALB/c mice infected with $L$. donovani or $L$. chagasi/L. infantum are the most widely studied models of VL, but they are also considered to be rather vulnerable. In the murine VL, C57BL/6 mice mount an early Th1 response, which prevents the further growth of the parasite, in turn producing a self-healing phenotype (Lehmann et al., 2000). In addition, the respective resistance and susceptibility of C57BL/6 and BALB/c strains depend not only on the Th1 and Th2 types of immune responses from $\mathrm{CD} 4^{+} \mathrm{T}$ cells, but also on the genetic background from the hosts (Afonso and Scott, 1993). For this reason, it is important to evaluate the immune response induced in inbred mouse strains. In this context, in the present study, spleen cells derived from naive mice were used to evaluate the in vitro immune stimulation, in order to select only the candidates that would be able to subvert the undesirable Th2 profile in a susceptible mouse strain.

Additionally, in vaccine studies, immunogens are administered in association with Th1-type adjuvants and, after only a few weeks, mice are infected and followed-up for about 2 or 3 months (Chávez-Fumagalli et al., 2010; Costa et al., 2014; Martins et al., 2013). In this time, spleen cells are collected and cultured in vitro with the antigens used in the immunization protocols, and/or with Leishmania extracts, in order to evaluate their immunogenicity. At this point, the levels of cytokines, such as IFN- $\gamma$ and IL-12, markers of the Th1 response, and IL- 4 and IL-10, indicators of Th2 response, are determined and, together with the results of the parasite burden, the efficacy of the immunogens is determined (Costa et al., 2014).

In relation to the protective efficiency, the present study described the efficacy of the association between an antigen derived from L. infantum, LiHyp1, which was recently shown as a protective antigen against this parasite species (Martins et al., 2013), and two adjuvant fractions, F2 and F4, in an attempt to verify if these compounds could, in fact, increase the immunogenicity of the rLiHyp1. The association between rLiHyp1 and both fractions was able to induce an in vivo Th1 response in the vaccinated animals, which was primed by production of high levels of IFN- $\gamma$, IL-12, and GM-CSF; as well as by low levels of IL-4 and IL-10. Additionally, a predominance of IgG2a isotype antibodies could be observed in these animals, facts that could have contributed to protecting the animals against the infection. More importantly, the F2 and F4 fractions presented similar results in relation to those vaccinated with saponin, although animals vaccinated with saponin have presented a slight to moderate inflammatory edema in their vaccinated footpads. This fact was not observed when F2 or F4 fractions were used with rLiHyp1 or administered alone. Immunization using the rLiHyp1 protein without an adjuvant was unable to induce protection against infection, emphasizing the need for the association of antigens with an effective adjuvant, as also described in other studies concerning other evaluated antigens (Coelho et al., 2003; Costa et al., 2014; Martins et al., 2013; Ramírez et al., 2014; Zanin et al., 2007).

The present study also demonstrates that the association between rLiHyp1 and F2 or F4 fractions in the vaccinated animals induced a decrease in the production of IL- 4 and IL-10. Very low levels of parasite-specific IL-10 were detected after the stimulation of spleen cells derived from vaccinated mice, 10 weeks after infection. By contrast, spleen cells from the control mice showed a significantly higher 
production of this cytokine. Indeed, the control of the parasitemediated IL-10 response may be critical for protection, since this cytokine is considered to be the most important factor for VL progression in mice treated with an anti-IL-10 receptor antibody (Murray et al., 2002), or after infection with Leishmania in IL-10 deficient mice (Murphy et al., 2001). In BALB/c mice, IgG1 levels are associated with disease progression into some Leishmania species, such as L. infantum (Martins et al., 2013) and L. amazonensis (Coelho et al., 2003). In the present study, immunized mice produced higher levels of Leishmania-specific IgG2a antibodies, as compared to IgG1 levels, also demonstrating the development of a Th1 immune response in these animals. As observed in the IFN- $\gamma$ and IL-12 production, spleen cells from vaccinated mice produced higher levels of GM-CSF, a cytokine related to macrophage activation and resistance in murine models against intracellular pathogens, such as L. infantum (Chávez-Fumagalli et al., 2010), L. major (Dumas et al., 2003), and L. donovani (Murray et al., 1995).

As regards the profile of $\mathrm{T}$ cells involved in IFN- $\gamma$ production, $\mathrm{CD}^{+} \mathrm{T}$ cells proved to be the major source of this cytokine in the protected mice, since the depletion of these cells in the cultures of spleen cells stimulated with SLA significantly diminished this response. Although previous reports have shown that the activation of both $\mathrm{CD} 4^{+}$and $\mathrm{CD}^{+}$cell subsets may be important for the killing of parasites in vaccinated mice using several parasite recombinant antigens (Chávez-Fumagalli et al., 2010; Costa et al., 2014; Martins et al., 2013), the present study's data suggest that $\mathrm{CD}^{+}$cells may contribute to a lesser extent to the induction of an IFN- $\gamma$-mediated response elicited by immunization using the rLiHyp1 protein, when the immunogen was evaluated in association with different adjuvants.

As a result of research into the development of vaccines against canine VL in the world, Brazil has become one of the countries in which commercial vaccines for the immunization of dogs are available. Two vaccines, Leishmune ${ }^{\circledR}$ (Parra et al., 2007) and Leish-Tec ${ }^{\circledR}$ (Fernandes et al., 2014), use saponin as an adjuvant in their compositions. Saponins, natural glycosides derived from steroid or triterpene, exhibited different biological and pharmacological activities. Notably, they can activate the mammalian immune system, which has led to their use as adjuvants. Their unique capacity to stimulate both the Th1 immune response and the production of cytotoxic T-lymphocytes makes the saponins ideal for use in vaccine compositions, in order to protect against intracellular pathogens, such as Leishmania (Rajput et al., 2007). Saponins have been used successfully for veterinary applications (Dalsgaard, 1987; Kensil et al., 1991), but are considered toxic for use in humans. In relation to their toxicity, saponins can cause local and granulomatous reactions; hemolysis, reflecting their affinity to cholesterol present in erythrocyte membranes; and local pain (Goldenthal et al., 1993; Kensil et al., 1991). As Agaricus blazei is considered as a nutraceutic, and its use is permitted in humans due to the absence of toxicity; and due to the fact that both F2 and F4 fractions were purified from this mushroom, without being associated with non-natural compounds, these products could well be considered for use in humans.

In conclusion, the results of the present study showed the purification and identification of Th1 adjuvants derived from Agaricus blazei, as well as their application in BALB/c mice, in association with the rLiHyp1 protein, to protect against VL. The associations between rLiHyp1/F2 and rLiHyp1/F4 induced a specific Th1 immune response, which was primed by production of high levels of IFN- $\gamma$, IL-12, and GM-CSF, as well as by low levels of IL-4 and IL-10. In addition, the immunogens proved to be protective against L. infantum. Therefore, it can be concluded that, in addition to their uses in other disease models, the F2 and F4 fractions could also be used as Th1 adjuvants in association with other antigens to protect against the Leishmania sp. infection.

\section{Acknowledgments}

This work was supported by grants from Pró-Reitoria de Pesquisa from UFMG (Edital 01/2014), Instituto Nacional de Ciência e Tecnologia em Nano-biofarmacêutica (INCT-Nanobiofar), FAPEMIG (CBB-APQ-00496-11 and CBB-APQ-00819-12), and CNPq (APQ472090/2011-9, RHAE-456287/2012-4, APQ-482976/2012-8 and APQ-488237/2013-0). MACF is a grant recipient of FAPEMIG/ CAPES. EAFC and MNM are grant recipient of CNPq.

\section{Appendix: Supplementary material}

Supplementary data to this article can be found online at doi:10.1016/j.exppara.2015.03.027.

\section{References}

Afonso, L.C., Scott, P., 1993. Immune responses associated with susceptibility of C57BL/10 mice to Leishmania amazonensis. Infect. Immun. 61, 2952-2959.

Ainsworth, E.A., Gillespie, K.M., 2007. Estimation of total phenolic content and other oxidation substrates in plant tissues using Folin-Ciocalteu reagent. Nat. Protoc. $2,875-877$

Al Tuwaijri, A.S., Mahmoud, A.A., Al Mofleh, I.A., Al Khuwaitir, S.A., 1987. Effect of glucan on Leishmania major infection in BALB/c mice. J. Med. Microbiol. 23, 363-365.

Alvar, J., Vélez, I.D., Bern, C., Herrero, M., Desjeux, P., Cano, J., et al., 2012. Leishmaniasis worldwide and global estimates of its incidence. PLoS ONE 7, e35671.

Badaró, R., Nascimento, C., Carvalho, J.S., Badaró, F., Russo, D., Ho, J.L., et al., 1994. Granulocyte-macrophage colony-stimulating factor in combination with pentavalent antimony for the treatment of visceral leishmaniasis. Eur. J. Clin. Microbiol. Infect. Dis. 13 (Suppl. 2), S23-S28.

Bernardshaw, S., Johnson, E., Hetlandy, G., 2005. An extract of the mushroom Agaricus blazei Murill administered orally protects against systemic Streptococcus pneumoniae infection in mice. Scand. J. Immunol. 62, 393-398.

Camelini, C.M., Maraschin, M., Mendonça, M.M., Zucco, C., Ferreira, A.G., Tavares, L.A., 2005. Structural characterization of beta-glucans of Agaricus brasiliensis in different stages of fruiting body maturity and their use in nutraceutical products. Biotechnol. Lett. 27, 1295-1299.

Carrión, J., Nieto, A., Iborra, S., Iniesta, V., Soto, M., Folgueira, C., et al., 2006. Immunohistological features of visceral leishmaniasis in BALB/c mice. Parasite Immunol. 28, 173-183.

Carvalho, V.M., Oliveira, G.D.C., Marques-da-Silva, E.A., Serafim, T.D., Afonso, L.C.C., Rezende, S.A., 2007. Successful vaccination against Leishmania chagasi infection in BALB/c mice with freeze-thaw Leishmania antigen and Corynabacterium parvum. Acta Trop. 104, 133-139.

Cerpa-Cruz, S., Paredes-Casillas, P., Landeros-Navarro, E., Bernard-Medina, A.G. Martínez-Bonilla, G., Gutiérrez-Ureña, S., 2013. Adverse events following immunization with vaccines containing adjuvants. Immunol. Res. 56, 299-303.

Chávez-Fumagalli, M.A., Costa, M.A., Oliveira, D.M., Ramírez, L., Costa, L.E., Duarte, M.C., et al., 2010. Vaccination with the Leishmania infantum ribosomal proteins induces protection in BALB/c mice against Leishmania chagasi and Leishmania amazonensis challenge. Microbes Infect. 12, 967-977.

Chen, L., Shao, H., 2006. Extract from Agaricus blazei Murill can enhance immune responses elicited by DNA vaccine against foot-and-mouth disease. Vet. Immunol. Immunopathol. 109, 177-182.

Coelho, E.A., Tavares, C.A., Carvalho, F.A., Chaves, K.F., Teixeira, K.N., Rodrigues, R.C., et al., 2003. Immune responses induced by the Leishmania (Leishmania) donovani A2 antigen, but not by the LACK antigen, are protective against experimental Leishmania (Leishmania) amazonensis infection. Infect. Immun. 71, 988-994.

Coler, R.N., Goto, Y., Bogatzki, L., Raman, V., Reed, S.G., 2007. Leish-111f, a recombinant polyprotein vaccine that protects against visceral Leishmaniasis by elicitation of $\mathrm{CD}^{+} \mathrm{T}$ cells. Infect. Immun. 75, 4648-4654.

Cook, J.A., Holbrook, T.W., Dougherty, W.J., 1982. Protective effect of glucan against visceral leishmaniasis in protective effect of glucan against visceral leishmaniasis in hamsters. Infect. Immun. 37, 1261-1269.

Costa, C.H.N., Peters, N.C., Maruyama, S.R., Brito, E.C., Santos, I.K.F.D.M., 2011. Vaccines for the leishmaniases: proposals for a research agenda. PLoS Negl. Trop. Dis. 5, e943.

Costa, L.E., Lima, M.I.S., Chávez-Fumagalli, M.A., Menezes-Souza, D., Martins, V.T., Duarte, M.C., et al., 2014. Subtractive phage display selection from canine visceral leishmaniasis identifies novel epitopes that mimic Leishmania infantum antigens with potential serodiagnosis applications. Clin. Vaccine Immunol. 21, 96-106.

Croft, S.L., Coombs, G.H., 2003. Leishmaniasis: current chemotherapy and recent advances in the search for novel drugs. Trends Parasitol. 19, 502-508.

Cui, L., Sun, Y., Xu, H., Xu, H., Cong, H., Liu, J., 2013. A polysaccharide isolated from Agaricus blazei Murill (ABP-AW1) as a potential Th1 immunity-stimulating adjuvant. Oncol. Lett. 6, 1039-1044.

Dalsgaard, K., 1987. Adjuvants. Vet. Immunol. Immunopathol. 17, 145-152.

Dubois, M., Gilles, K.A., Hamilton, J.K., Rebers, P.A., Smith, F., 1956. Colorimetric method for determination of sugars and related substances. Anal. Chem. 28, 350-356. 
Dumas, C., Muyombwe, A., Roy, G., Matte, C., Ouellette, M., Olivier, M., et al., 2003. Recombinant Leishmania major secreting biologically active granulocytemacrophage colony-stimulating factor survives poorly in macrophages in vitro and delays disease development in mice. Infect. Immun. 71, 6499-6509.

Engel, A.L., Sun, G.C., Gad, E., Rastetter, L.R., Strobe, K., Yang, Y., et al., 2013. Protein-bound polysaccharide activates dendritic cells and enhances OVA-specific T cell response as vaccine adjuvant. Immunobiology 218, 1468-1476.

Fernandes, A.P., Coelho, E.A.F., Machado-Coelho, G.L.L., Grimaldi, G., Jr., Gazzinelli, R.T., 2012. Making an anti-amastigote vaccine for visceral leishmaniasis: rational, update and perspectives. Curr. Opin. Microbiol. 15, 1-10.

Fernandes, C.B., Junior, J.T.M., Jesus, C., Souza, B.M.P.D.S., Laranjeira, D.F., Fraga, D.B.M., et al., 2014. Comparison of two commercial vaccines against visceral leishmaniasis in dogs from endemic areas: IgG, and subclasses, parasitism, and parasite transmission by xenodiagnosis. Vaccine 32, 1287-1295.

Fox, C.B., Kramer, R.M., Barnes, V.L., Dowling, Q.M., Vedvick, T.S., 2013. Working together: interactions between vaccine antigens and adjuvants. Ther. Adv. Vaccines 1, 7-20.

Giunchetti, R.C., Correa-Oliveira, R., Martins-Filho, O.A., Teixeira-Cervalho, A., Roalt, B.M., Oliveira, A.S.R.D., et al., 2007. Immunogenicity of a killed Leishmania vaccine with saponin adjuvant in dogs. Vaccine 25, 7674-7686.

Goldenthal, K.L., Cavagnaro, J.A., Alving, C.R., Vogel, F.R., 1993. National cooperative vaccine development working group. Safety evaluation of vaccine adjuvants. AIDS Res. Hum. Retroviruses 9, S45-S49.

Gonçalves, J.L., Roma, E.H., Gomes-Santos, A.C., Aguilar, E.C., Cisalpino, D., Fernandes, L.R., et al., 2012. Pro-inflammatory effects of the mushroom Agaricus blazei and its consequences on atherosclerosis development. Eur. J. Nutr. 51, 927-937.

Handman, E., 2001. Leishmaniasis: current status of vaccine development. Clin. Microbiol. Rev. 14, 229-243.

Holbrook, T.W., Cook, J.A., Parker, B.W., 1981. Immunization against Leishmania donovani: glucan as an adjuvant with killed promastigotes. Am. J. Trop. Med. Hyg. 30, 762-768.

Kahl, L.P., Scott, C.A., Leichuk, R., Gregoriadis, G., Liew, R.Y., 1989. Vaccination against murine cutaneous leishmaniasis by using Leishmania major antigen/liposomes. Optimization and assessment of the requirement for intravenous immunization. J. Immunol. 142, 4441-4449.

Kensil, C.R., Patel, U., Lennick, M., Marciani, D., 1991. Separation and characterization of saponins with adjuvant activity from Quillaja-saponaria molina cortex. J. Immunol. 146, 431-437.

Koike, Y., Yoo, Y.C., Mitobe, M., Oka, T., Okuma, K., Tono-Oka, S., et al., 1998. Enhancing activity of mycobacterial cell-derived adjuvants on immunogenicity of recombinant human hepatitis B virus vaccine. Vaccine 16, 1982-1989.

Lee, B.Y., Bacon, K.M., Shah, M., Kitchen, S.B., Connor, D.L., Slayton, R.B., 2012. The economic value of a visceral leishmaniasis vaccine in Bihar state, India. Am. J. Trop. Med. Hyg. 86, 417-425.

Lehmann, J., Enssle, K.H., Lehmann, I., Emmendorfer, A., Lohmann-Matthes, M.L., 2000. The capacity to produce IFN-gamma rather than the presence of interleukin-4 determines the resistance and the degree of susceptibility to Leishmania donovani infection in mice. J. Interferon Cytokine Res. 20, 63-77.

Lowry, O.H., Rosebrough, N.J., Farr, L., Randall, R.J., 1951. Protein measurement with the Folin phenol reagent. J. Biol. Chem. 193, 265-275.

Maehara, Y., Tsujitani, S., Saeki, H., Oki, E., Yoshinaga, K., Emi, Y., et al., 2012. Biological mechanism and clinical effect of protein-bound polysaccharide K (Krestin ${ }^{\circledR}$ ): review of development and future perspectives. Surg. Today 42, 8-28.

Martins, V.T., Chávez-Fumagalli, M.A., Costa, L.E., Canavaci, A.M.C., Martins, A.M.C.C., Lage, P.S., et al., 2013. Antigenicity and protective efficacy of a Leishmania amastigote-specific protein, member of the super-oxygenase family, against visceral leishmaniasis. PLoS Negl. Trop. Dis. 7, e2148.

Meng, Y.Z., Caijiao, W., HuSheng, Z., XianWu, P., JianMin, F., 2008. Protective effect of polysaccharides from Morinda officinalis on bone loss in ovariectomized rats. Int. J. Biol. Macromol. 43, 276-278.

Minodier, P., Parola, P., 2010. Cutaneous leishmaniasis treatment. Travel Med. Infect. Dis. 5, 150-158.

Misra, A., Dube, A., Srivastava, B., Sharma, P., Srivastava, J.K., Katiyar, J.C., et al., 2001. Successful vaccination against Leishmania donovani infection in Indian langur using alum-precipitated autoclaved Leishmania major with BCG. Vaccine 19, 3485-3492.

Murphy, M.L., Wille, U., Villegas, E.N., Hunter, C.A., Farrell, J.P., 2001. IL-10 mediates susceptibility to Leishmania donovani infection. Eur. J. Immunol. 31, 2848-2856.

Murray, H.W., Cervia, J.S., Hariprashad, J., Taylor, A.P., Stoeckle, M.Y., Hockman, H., 1995. Effect of granulocyte-macrophage colony-stimulating factor in experimental visceral leishmaniasis. J. Clin. Invest. 95, 1183-1192.
Murray, H.W., Lu, C.M., Mauze, S., Freeman, S., Moreira, A.L., Kaplan, G., et al., 2002. Interleukin-10 (IL-10) in experimental visceral leishmaniasis and IL-10 receptor blockade as immunotherapy. Infect. Immun. 70, 6284-6293.

Obaid, K.A., Ahmad, S., Khan, H.M., Mahdi, A.A., Khanna, R., 1989. Protective effect of $L$. donovani antigens using glucan as an adjuvant. Int. J. Immunopharmacol. $11,229-235$.

O'Hagan, D.T., de Gregorio, E., 2009. The path to a successful vaccine adjuvant: "the long and winding road". Drug Discov. Today 14, 541-551.

Oliveira, L.F., Schubach, A.O., Martins, M.M., Passos, S.L., Oliveira, R.V., Marzochi, M.C., et al., 2011. Systematic review of the adverse effects of cutaneous leishmaniasis treatment in the New World. Acta Trop. 118, 87-96.

Ooi, V.E., Liu, F., 2000. Immunomodulation and anti-cancer activity of polysaccharideprotein complexes. Curr. Med. Chem. 7, 715-729.

Panaro, M.A., Acquafredda, A., Lisi, S., Lofrumento, D.D., Mitolo, V., Sisto, M., et al., 2001. Nitric oxide production by macrophages of dogs vaccinated with killed Leishmania infantum promastigotes. Comp. Immunol. Microbiol. Infect. Dis. 24 187-195.

Parra, L.E., Borja-Cabrera, G.P., Santos, F.N., Souza, L.O.P., Palatnik-de-Sousa, C.B., Menz I., 2007. Safety trial using the Leishmune ${ }^{\circledR}$ vaccine against canine visceral leishmaniasis in Brazil. Vaccine 25, 2180-2186.

Petrovsky, N., Aguilar, J.C., 2004. Vaccine adjuvants: current state and future trends. Immunol. Cell Biol. 82, 488-496.

Petrovsky, N., Cooper, P.D., 2011. Carbohydrate-based immune adjuvants. Expert Rev. Vaccines 10, 523-537.

Pillemer, L., Ecker, E.E., 1941. Anticomplementary factor in fresh yeast. J. Biol. Chem. $137,139-142$.

Rajput, Z.I., Hu, S., Xiao, C., Arijo, A.G., 2007. Adjuvant effects of saponins on animal immune responses. J. Zhejiang Univ. Sci. B 8, 153-161.

Ramírez, L., Santos, D.M., Souza, A.P., Coelho, E.A.F., Barral, A., Alonso, C., et al., 2013. Evaluation of immune responses and analysis of the effect of vaccination of the Leishmania major recombinant ribosomal proteins L3 or L5 in two different murine models of cutaneous leishmaniasis. Vaccine 7, 3.

Ramírez, L., Villen, L.C., Duarte, M.C., Chávez-Fumagalli, M.A., Valadares, D.G., Santos, D.M., et al., 2014. Cross-protective effect of a combined L5 plus L3 Leishmania major ribosomal protein based vaccine combined with a Th1 adjuvant in murine cutaneous and visceral leishmaniasis. Parasit. Vectors 7, 3-10.

Ready, P.D., 2014. Epidemiology of visceral leishmaniasis. Clin. Epidemiol. 6, 147-154.

Reed, S.G., Orr, M.T., Fox, C.B., 2013. Key roles of adjuvants in modern vaccines. Nat. Med. 19, 1597-1608.

Stacey, K.J., Blackwell, J.M., 1999. Immunostimulatory DNA as an adjuvant in vaccination against Leishmania major. Infect. Immun. 67, 3719-3726.

Tonui, W.K., Mejia, J.S., Hochberg, L., Mbow, M.L., Ryan, J.R., Chan, A.S., et al., 2004 Immunization with Leishmania major exogenous antigens protects susceptible $\mathrm{BALB} / \mathrm{c}$ mice against challenge infection with Leishmania major. Infect. Immun. 72, 5654-5661.

Valadares, D.G., Duarte, M.C., Oliveira, J.S., Martins, V.T., Costa, L.E., Leite, J.P.V., et al., 2011. Leishmanicidal activity of the Agaricus blazei Murill in different Leishmania species. Parasitol. Intern. 60, 357-363.

Valadares, D.G., Duarte, M.C., Ramírez, L., Chávez-Fumagalli, M.A., Lage, P.S., Martins, V.T., et al., 2012a. Therapeutic efficacy induced by the oral administration of Agaricus blazei Murill against Leishmania amazonensis. Parasitol. Res. 113 $1-10$.

Valadares, D.G., Duarte, M.C., Ramírez, L., Chávez-Fumagalli, M.A., Martins, V.T., Costa, L.E., et al., 2012b. Prophylactic or therapeutic administration of Agaricus blazei Murill is effective in treatment of murine visceral leishmaniasis. Exp. Parasitol. $132,228-236$.

Wang, J., Dong, S., Liu, C., Wang, W., Sun, S., Gu, J., et al., 2010. beta-Glucan oligosaccharide enhances CD8(+) T cells immune response induced by a DNA vaccine encoding hepatitis B virus core antigen. J. Biomed. Biotechnol. 645213.

World Health Organization, 2010. Control of the leishmaniases: report of a meeting of the 399 WHO Expert Committee on the Control of Leishmaniases. <http://whqlibdoc.who.int/trs/WHO_TRS_949_eng.pdf>.

Yatawara, L., Wickramasinghe, S., Nagataki, M., Takamoto, M., Nomura, H., Ikeue, Y., et al., 2009. Aureobasidium-derived soluble branched (1,3-1,6) beta-glucan (Sophy beta-glucan) enhances natural killer activity in Leishmania amazonensis-infected mice. Korean J. Parasitol. 47, 345-351.

Zanin, F.H., Coelho, E.A., Tavares, C.A., Marques-da-Silva, E.A., Costa, M.M.S., Rezende, S.A., et al., 2007. Evaluation of immune responses and protection induced by A2 and nucleoside hydrolase (NH) DNA vaccines against Leishmania chagasi and Leishmania amazonensis experimental infections. Microbes Infect. 9, 10701077. 